\title{
FEM-BASED MODELLING OF ELASTIC PROPERTIES AND ANISOTROPIC SINTER SHRINKAGE OF METAL EAM
}

\author{
Rosnitschek, T.; Hueter, F. \& Alber-Laukant, B. \\ Chair of Engineering Design and CAD, University of Bayreuth, Universitaetsstr. 30, 95447 Bayreuth, \\ Germany \\ E-Mail: tobias.rosnitschek@uni-bayreuth.de
}

\begin{abstract}
The fabrication of nearly fully dense metal parts via additive material extrusion processes is an auspicious alternative to powder-bed-based methods. After the extrusion of the material, the parts are debindered and sintered. Due to the process, the shrinkage of the parts and the material behaviour is orthotropic which causes problems to obtain the desired dimensional accuracy. Classical methods for simulating the sinter process are complex and demand the knowledge of various material parameters that have to be determined experimentally. This paper discusses analytical and numerical methods for predicting the effective properties of additively fabricated parts and presents a new and simple approach for the prediction of shrinkage, warpage and internal stresses caused by the sintering process based on a thermomechanical finite element analysis. The presented framework can be used to model the material behaviour without the need of extensive experimental data and is adaptable to various machines and materials. The proposed approach is exemplary shown. The results validate its functionality to predict sinter induced shrinkage and warpage as well as the locations of maximum internal stresses.

(Received in February 2020, accepted in April 2020. This paper was with the authors 1 week for 1 revision.)
\end{abstract}

Key Words: FEA, Anisotropy Shrinkage, Sintering, Material Extrusion Additive Manufacturing, Metallic Components, Representative Volume Elements (RVE)

\section{INTRODUCTION}

Additive manufacturing (AM) is on the edge from a technology mainly used in rapid prototyping towards the so-called physical arm of industrial digitalisation. It enables a much higher freedom of design compared to conventional manufacturing techniques [1-3]. Furthermore, as a more efficient use of resources and better environmental performance is desirable for the development of new products $[4,5]$, AM is an auspicious option to fulfil these objectives [1-6].

Predominant processes for the fabrication of metallic parts rely on high-energy beams such as laser or electron beams used for the bonding of particles in a powder bed. Thus, the machines require inert gas recirculating filtering equipment and chilling systems, which is why the implementation of such processes is affiliated with high investment costs [7, 8]. Additionally, these processes imply safety concerns, which arise from the use of the fine metal powder [9]. As the urge for economical alternatives is rising especially in small businesses and university laboratories [10], the use of plastic-metal composites for extrusion based additive manufacturing (EAM) with subsequent debindering and sintering processes is a very promising approach to ease the entry into metal AM [11].

\subsection{Extrusion based additive manufacturing of metallic components}

EAM processes in general and the fused filament fabrication (FFF) of thermoplastic polymers in particular are by far the most common AM techniques. These processes are based on softening the material and pushing it through an orifice to fabricate layers which build a threedimensional structure [7, 12]. Equipment for EAM is rather inexpensive and easy to operate 
compared to other processes. The combination of the FFF process with subsequent debinding and sintering was primary referred to as FDMet. The complete process is dividable into the three steps of shaping, debinding and sintering (SDS) $[8,13]$ as illustrated in Fig. 1.

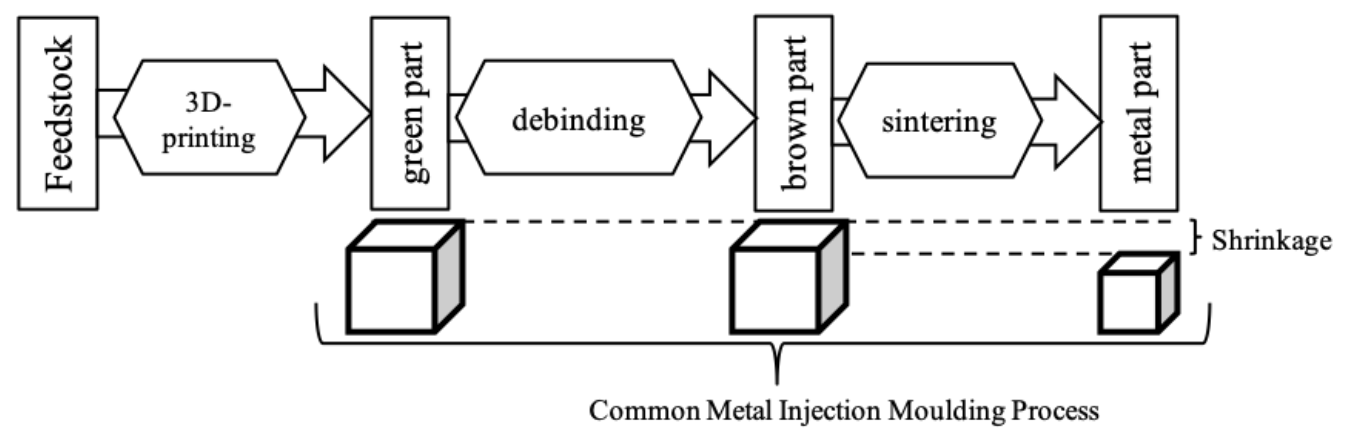

Figure 1: Schematic overview of the SDS process.

In the first step, a three-dimensional green part is printed by extruding the raw material through an orifice. The subsequent debinding step removes the binding agent nearly completely from the part, which is now referred to as brown part. The final sintering process comprises a thermal treatment near the melting point of the powder's major constituent. Rearrangement, particle movement and mass transportation occur during this step [7] causing a densification of the part. Multiple diffusion processes are involved in the sintering process reducing the surface energy of the highly porous brown part. At its final stage the density increases up to $99 \%$ of its theoretical value. This step is crucial in understanding the shrinkage of the parts, which is subject of the following section.

The material feedstock used is quite similar to the feedstocks used in powder injection moulding (PIM) [8]. It is obvious that the filament has to fulfil certain requirements, like appropriate viscous properties, to be manufacturable on the equipment. Typical feedstocks used have a volume fraction of binding systems ranging from 35 to 65 vol. \% [7, 8, 10, 12-15].

The debinding process removes the binding agent almost completely [8] leaving a highly porous brown part. During the sintering step shrinkage of 35 to $65 \mathrm{vol}$. \% occurs, which causes issues regarding the geometrical accuracy of the sintered parts. It is shown in various studies, that the shrinkage is orthotropic $[10,11,14]$ partially due to the characteristics of the FFF process. This anisotropic effect causes additional issues in predicting the correct "out of shape" green-part. It is emphasised in [7] that the shrinkage has to be controlled to be competitive with other AM-techniques. However, problems with distortion due to shrinkage are also known from conventional manufacturing processes such as injection moulding, which are influenced, for instance, by the wall temperature or fractions of recycled material in the starting feedstock [16]. However, since PIM or IM in general is used for very high volumes, it is easy to perform tests to determine shrinkage, whereas this is not a reasonable approach for the small volumes of AM production. Thus, it is of high interest to predict the occurring shrinkage and warpage of the part in an efficient and precise manner to push this technology further.

\subsection{Simulation of sinter shrinkage: state of the art}

It is one of the main goals of sinter simulation to determine the non-uniform shrinkage of the parts. Although sintering is a commonly used industrial process, its modelling is still a challenging task [17]. The sintering process in general consists of at least six different mass transport mechanisms, most of them diffusion controlled, which contribute to the bonding between particles and the growth of necks between them [12]. Hence, this multitude of involved processes makes it difficult to account to all of them in an appropriate manner within 
the numerical simulation. Primarily phenomenological approaches are used to describe the sintering process [17-20]. These models are based on examples from PIM related research on ceramic or metallic components. It is to emphasise that only models for solid phase sintering are of interest for the sinter simulation of EAM metal parts. Methodologies to model liquid phase sintering, as shown in [21], can be neglected.

In detail, the sintering process is dividable into three stages: heating-phase, sintering and cooling-phase [20] as illustrated in Fig. 2.

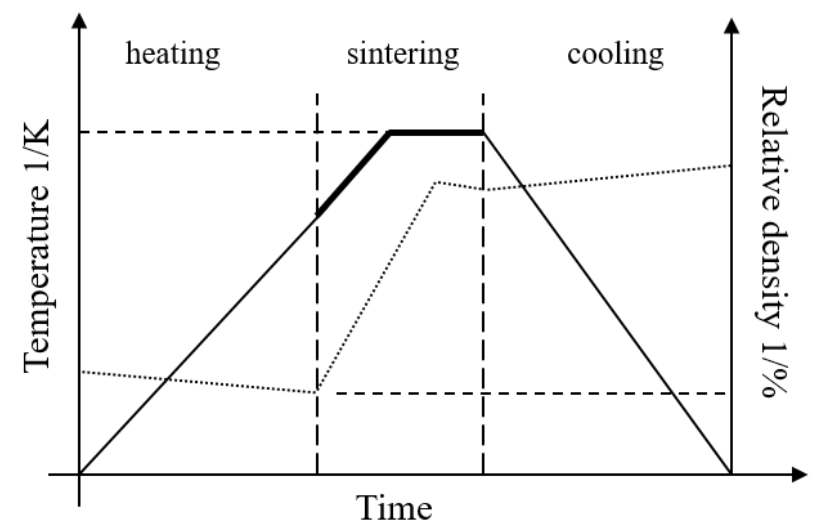

Figure 2: Schematically sinter curve and relative density evolution, following [20].

On this empirical basis, the begin of a sintering process is marked by a thermal expansion due to the heating of part, as sintering is strongly depending on the temperature, leading to a reduction of the relative density. After sintering starts, the changes of the internal structure dominate causing an increase of the relative density. The reduction of the free surface energy is the driving force of the process. Its activity and therefore the densification rate are gradually dismantled with the ongoing sinter process. After the sintering has finished, the cooling causes thermal contraction, leading to a small increase of the relative density.

Addressing these phenomena, it can be stated that the material behaviour is mainly influenced by change of the relative density due to changes of the internal structure, and by temperature. Previous approaches used elastic viscoplastic models for the description of the sintering induced and thermal shrinkage [17-20]. In this case, the total deformation gradient, is decomposable as

$$
\boldsymbol{F}=\boldsymbol{F}^{m} \boldsymbol{F}^{i m}
$$

with $\boldsymbol{F}^{m}$ being the mechanical and $\boldsymbol{F}^{i m}$ the non-mechanical component [20]. The latter is assumed solely dependent on the volumetric changes caused by thermal expansion and sintering. Thus, the non-mechanical component can be expressed by the time dependent rate of change in the thermal strain $\dot{\varepsilon}^{t}$ and the time rate of change in the sintering strain $\dot{\varepsilon}^{s}$ [20]

$$
\begin{gathered}
\boldsymbol{F}^{i m}=\left(1+\dot{\varepsilon}^{t}+\dot{\varepsilon}^{s}(T)\right) \mathbf{1} \\
\dot{\varepsilon}^{t}=\alpha_{T} \dot{T} \\
\dot{\varepsilon}^{s}(T)=-\frac{1}{3}\left(\frac{\dot{\rho}}{\rho}\right)
\end{gathered}
$$

The thermal strain rate is characterised by the linear coefficient of thermal expansion $\alpha_{T}$, which is assumed to be constant. The densification rate $\dot{\rho}$ controls the time rate change in the sintering strain. The sign of the equation indicates shrinkage with increasing mass density [20] and the factor $1 / 3$ assumes an isotropic densification of the part.

The material behaviour mainly depends on the change of density. A relative density is introduced in [20] as a measure of densification: 


$$
\rho_{\text {rel }}(T(t))=\frac{\rho(T(t))}{\rho_{s}}
$$

Here $\rho$ and $\rho_{s}$ are the densities at the current and the final stage of sintering respectively. The current density depends on the thermal history. It is necessary to carry out thermomechanical analysis to obtain the density for a specific thermal history $T(t)$.

For the isotropic case, the relative volumetric change can be expressed by the Jacobian $J(T)$ based on the specimen's height [20]:

$$
J(T)=\left(\frac{h_{i}+\delta h(T)}{h_{i}}\right)^{3}
$$

Here, $h_{i}$ describes the initial height of the specimen and $\delta h(T)$ is the continually measured height change. Since the Jacobian is basically the relative volume change of the part during sintering, the current density $\rho$ can be written as a function of the temperature dependent height change $\delta h(T)$ and the initial density $\rho_{i}[20]$ :

$$
\rho(\delta h(T))=\frac{\rho_{i}}{J(T)}=\rho_{i}\left(\frac{h_{i}}{h_{i}+\delta h(T)}\right)^{3}
$$

This leads to a time-evolution equation for sintering induced strain $\dot{\varepsilon}^{s}[20]$ :

$$
\dot{\varepsilon}^{s}(T, \delta h)=-\frac{1}{3} \Omega(T)\left[\rho_{r e l}^{\infty}(T)-\rho_{r e l}(T)\right]^{n(T)}
$$

The parameters $\Omega(T)$ and $n(T)$ are temperature dependent material parameters. Variable $\rho_{\text {rel }}^{\infty}$ is a stationary value of the relative density under a certain isothermal environment at a specific temperature, also referred to as ultimate relative density [20]. All of these parameters have to be determined experimentally and a thermomechanical analysis is inevitable for setting up the model.

An alternative model is described in [17] based on the approach in [18]. The total strain rate is subdivided into a thermal strain as an elastic and viscoplastic strain rate. The relationship for the total strain rate as described by [17] can be written as

$$
\dot{\varepsilon}=\dot{\varepsilon}_{t h}+\dot{\varepsilon}_{e}+\dot{\varepsilon}_{v p}=\alpha \Delta \dot{T} I+C_{e} \dot{\sigma}+\frac{\operatorname{dev}(\sigma)}{2 G}+\frac{\sigma_{m}-\sigma_{s}}{3 K} I
$$

where $C_{e}$ is the elastic compliance matrix, $I$ is the second order identify tensor, $\sigma_{m}=\operatorname{tr}(\sigma) / 3$ is the trace of the stress tensor, $G$ is the shear viscosity modulus, $K$ is the bulk viscosity modulus and $\sigma_{s}$ is the sintering stress. Again, it is inevitable to conduct various dilatometry experiments to determine the parameters $K, G, \sigma_{s}$.

Both models described above require various material parameters, which have to be determined experimentally. This is a considerable drawback, because experimental data acquisition is time consuming and expensive and one needs access to appropriate laboratories.

To establish the EAM for metal parts as an easy to applicate technique it is necessary to develop an efficient simulation model to predict the shape and the distortion of the printed and sintered parts without the need of extensive experiments to calibrate the simulation model. Thus, it is the objective of this study to provide an easy to use method to predict the shape and the internal stresses of the final parts to support researchers and product designers working on this process.

\section{MODELLING}

The mesostructure of parts from the shaping process of FDM is different to that from conventional PIM. Usually, the parts from conventional PIM are uniformly compacted before sintering to avoid voids inside the green part. As 3D-printed parts are difficult to compact in traditional machines, due to their mostly complex geometry, the green-parts show less relative 
density than the conventional compacted ones. Consequently, the sintering induced strains are larger, and the shrinkage is anisotropic because of the layer wise building process during 3D printing. As all of the simulation models described above assume isotropic behaviour for the derivation of the constitutive equation, adaptions are required to capture the anisotropic material behaviour. Additionally, it is crucial to reduce the number of required material parameters for calibrating the model. In the following, we present a new modelling strategy based on a virtual thermomechanical approach to model the sinter process phenomenologically. Within this approach, the sintering process is modelled as a transient thermomechanical process. Each virtual temperature state represents a specific stage of the sintering process. The sinter strain and thereby the densification is described as a virtual thermal contraction within this model.

\subsection{Framework}

The sintering process is mainly dividable into the sinter-induced shrinkage and thermal shrinkage. In order to avoid the need of viscoelastic constants in this methodology, the whole effect of volume change during the sintering is summarised in a virtual equivalent total thermal strain, which is induced to the part via a virtual temperature field $T_{v}$. The orthotropic behaviour of the material is considered and characterised by its total shrinkage in the different directions, which are depicted in Fig. 3, where the xy-plane describes the printing plane and $\mathrm{z}$ is the stacking direction.

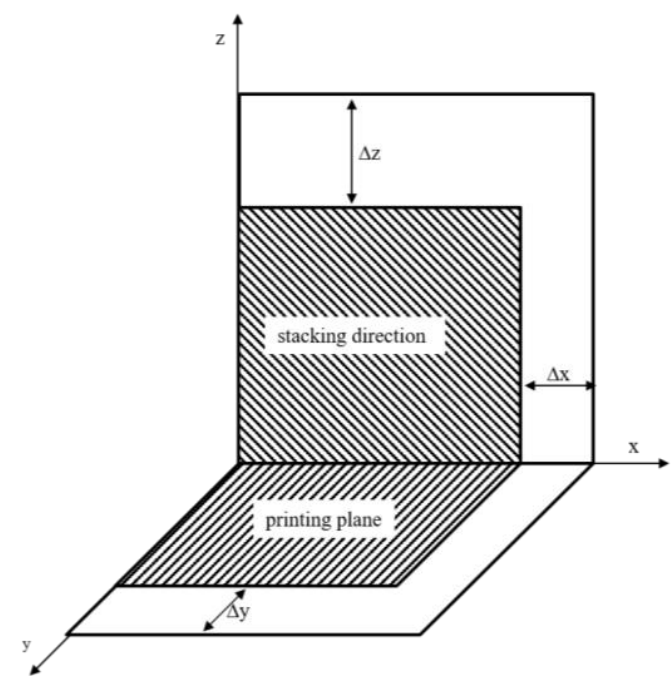

Figure 3: Shrinkage behaviour of MFF parts with its aligned layers of interest.

Following this approach, the relative density of this orthotropic metallic material is obtained analogously to Eq. (7) by

$$
\frac{\rho\left(\delta h\left(T_{v}\right)\right)}{\rho_{i}}=\frac{1}{J(T)}=\left(\frac{h_{i, x}}{h_{i, x}+\delta h_{x}\left(T_{v}\right)}\right) \cdot\left(\frac{h_{i, y}}{h_{i, y}+\delta h_{y}\left(T_{v}\right)}\right) \cdot\left(\frac{h_{i, z}}{h_{i, z}+\delta h_{z}\left(T_{v}\right)}\right)
$$

with $h_{i, j}$ being the initial height in each direction and $\delta h_{j}\left(T_{v}\right)$ its aligned incremental change. Since a virtual temperature field is used to induce the sintering shrinkage via a fictitious thermal contraction, the change in density can be expressed with help of equivalent virtual thermal expansion coefficients defined as

$$
\alpha_{j}=\frac{\delta h_{j}}{h_{i, j}} \frac{1}{\Delta T_{v}}
$$

where $\Delta T_{v}$ is the virtual temperature gradient. The virtual thermal strain, which represents the equivalent total sinter strain, is thereby defined for each direction as 


$$
\varepsilon_{j}=\alpha_{j} \Delta T_{v}=\frac{\delta h_{j}}{h_{i, j}}
$$

The coefficients of virtual thermal expansion are further referred to as shrinkage parameters, since they represent the relative contraction during the sintering process in each direction. By virtue of Eq. (12), the relative density can be expressed as

$$
\frac{\rho\left(T_{v}\right)}{\rho_{i}}=\frac{1}{\left(1+\alpha_{x} \Delta T_{v}\right) \cdot\left(1+\alpha_{y} \Delta T_{v}\right) \cdot\left(1+\alpha_{z} \Delta T_{v}\right)}=\frac{1}{\left(1+\alpha_{p} \Delta T_{v}\right)^{2} \cdot\left(1+\alpha_{s} \Delta T_{v}\right)}
$$

Since the shrinkage in $\mathrm{x}$ - and $\mathrm{y}$-direction is assumed to be identical $[10,12]$, only two input parameters $\alpha_{p}$ and $\alpha_{s}$ have to be determined via Eq. (13) and hence the lengths of the green and the sintered part need to be measured beforehand, which define the total shrinkage of the part. Additionally, a few material data of the bulk material are needed for modelling; an overview of all required input parameters and their origin is given in Table I.

Table I: Input parameters.

\begin{tabular}{|c|l|}
\hline Parameter & \multicolumn{1}{|c|}{ Meaning } \\
\hline$E_{0}$ & Elastic modulus (Bulk) \\
\hline$v_{0}$ & Poisson ratio (Bulk) \\
\hline$\rho_{0}$ & Density (Bulk) \\
\hline$B A$ & Volume fraction binding agent (Data sheet) \\
\hline$\alpha_{p}$ & In plane shrinkage parameter (x, y) (Measurement) \\
\hline$\alpha_{s}$ & Shrinkage parameter in stacking direction (z) (Measurement) \\
\hline
\end{tabular}

It is assumed that the sintered body has reached its theoretical maximum density, if the shrinkage equals the volume fraction of the binding agent $(B A)$ in the filament, which is known form the datasheet of the filament. The ratio of the final shrinkage $\Delta V$ and the maximum shrinkage $\Delta V_{\infty}$ is therefore given by

$$
\frac{\Delta V}{\Delta V_{\infty}}=\frac{1-\left(1+\alpha_{p} \Delta T_{v}\right)^{2}\left(1+\alpha_{s} \Delta T_{v}\right)}{B A}
$$

This approach assumes that the printing process is optimal, and the parts fabricated without any artefacts. Therefore, the presented approach can be used to describe the material behaviour without the need of extensive experimental data.

\subsection{Material behaviour}

Aside from the shrinkage, the orientation of the printing path strongly influences the mechanical behaviour as described in [22]. Therefore, the 3D-printed material shows characteristics comparable to fibre-reinforced polymers in a distinct manner. The modelling of this anisotropic material behaviour is crucial for the design.

As described in the literature [12, 17-20], the volume contraction is mainly caused by the densification during the sintering process, leading to a decreasing porosity within the part. As the porosity affects the elastic properties, i.e. the elastic modulus and the Poisson's ratio, a constitutive equation is needed relating these physical quantities for the SDS application. The influence of pores on the elastic material behaviour has been studied widely in literature, and numerous models have been derived [23-27]. Choren et al. [26] studied this subject for AM applications considering voids.

In this section, we describe an empirical and a numerical approach for setting the material data and compare them further on.

As the task is to describe a porosity range of about $45-2 \%$, the empirical Ramakrishnan and Arunachalam (RK) model [24, 26] is applied, which is said to satisfy all limiting 
conditions of zero porosity and a totally porous solid. The proposed relationship addresses porous solids with randomly distributed spherical voids [26]

$$
E_{p}=E_{0} \frac{(1-P)^{2}}{\left(1+\left(2-3 v_{p}\right) P\right)}
$$

where $E_{i}$ address the porous and the bulk elastic modulus respectively. $P$ denotes the porosity and $v$ the Poisson's ratio. Following [24], the Poisson ratio varies with the density according to the commonly chosen phenomenological approach given in Eq. (16) [17]

$$
v_{p} \approx v_{0} \sqrt{\frac{\rho}{3-2 \rho}}
$$

The current density may be calculated via Eq. (13) in this context. Eq. (17) gives a definition of the current porosity $P$ during sintering

$$
P=1-\frac{\rho\left(T_{v}\right)}{\rho_{0}} .
$$

Adversely to this model is its isotropic formulation, as the porosity in the printed greenparts is not characterised by randomly distributed spherical voids, but by periodically arranged voids of arbitrary form due to the FFF-process. At this point, the RK model becomes insufficient, as it does not properly address anisotropy.

In [22] $\mu$-CT pictures are shown, which proof that the inner structure of the printed and sintered parts is dominated by connected pore channels between the printing traces. Therefore, it is appropriate to model the material as a 'fibre-reinforced composite', where the 'fibre' is actually a channel-shaped cavity. In order to gather the anisotropic behaviour, representative volume elements (RVE) are used. A representative section of the microstructure is considered to characterise the effective behaviour of the material on the macroscopic scale. Based on the results from micro-tomography shown in [22], the RVE consists of a channel pore inside of a cuboidal steel matrix. We assume that the connected pore channels are predominant for the mechanical properties and other voids are negligible. The geometry of the RVE is chosen based on layer height and hatching distance, their ratio is further used to determine the pore channel's cross-sectional area. Both uniaxial tension and pure shear load are applied in order to determine the global elastic properties of the RVE. In order to prohibit unrealistic deformation of the lateral surfaces, periodic boundary conditions are imposed on the RVE's boundaries using linear constraint equations. Fig. 4 depicts the FE models including the boundary conditions for both load cases.
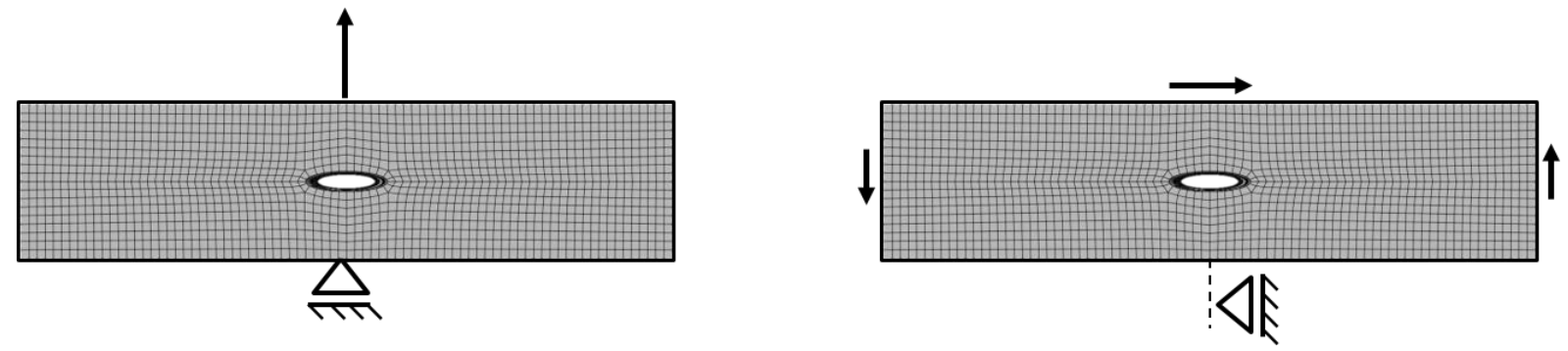

Figure 4: FE-Models of the RVE for tension (left) and shear load (right).

The boundary conditions and constraints used were imposed on an isotropic solid cubic RVE beforehand to validate their correctness. The resulting elastic modulus and Poisson's ratio from the simulation matched the assigned values exactly, confirming the correctness of the model setup.

In contrast to the transversal isotropic nature of shrinkage, the global elastic properties of the RVE are fully orthotropic leading to nine different elastic constants, one elastic modulus, 
Poisson's ratio and shear modulus in each direction. Hence, the boundary conditions and constraints are rotated to gain the material response in all three directions.

The porosity is varied in nine discrete steps and the material parameters obtained are interpolated between these points using cubic splines. The effective material parameters resulting from the RK model and the RVE simulation are shown in Fig. 6. The results are compared and discussed in section 3.1 below.

\subsection{FEA modelling}

A transient thermomechanical simulation is set up for modelling the sinter process. Since this simulation of an equivalent, thermal induced shrinkage is just a simple analogous model for the physical sinter process, thermal material parameters such as conductivity and heat capacity have no obvious physical meaning and can be chosen in a manner to achieve good numerical convergence. Here, the choice of the thermal material parameters aims for a homogenous temperature distribution inside the part at each time step. The Fourier number is an appropriate dimensionless quantity to control the transient heat transport process. It relates the duration of the transient cooling process to the heat transportation rate. Here, the Fourier number has no physical meaning and thus, is used to ensure numerical stability and a homogeneous temperature field. Therefore, a high Fourier number is chosen, so that the heat is transported away rapidly.

A virtual temperature range from $100 \mathrm{~K}$ to $0 \mathrm{~K}$ covers the complete sintering process. At the beginning of the simulation, the part has a homogeneous virtual temperature of $100 \mathrm{~K}$. This state corresponds to the transition phase of the sintering process with all of the binding agent removed and the first sinter necks grown. At this time, the porosity is at its maximum. A virtual temperature of $0 \mathrm{~K}$ represents the final sintered and cooled state with minimal porosity.

For the shrinkage, $\alpha_{p}$ and $\alpha_{s}$ are used as orthotropic coefficients of thermal expansion. The cooling of the part is enforced by a convective surface boundary condition, regulated by a film coefficient on the part's surface and an ambient temperature of $0 \mathrm{~K}$. On the mechanical side, the boundary conditions are chosen so that the part is statically determined and its contraction unconstrained. Additionally, gravity loading is applied to the part. Abaqus 2018 Hotfix 4 from Dassault Systems is used for the simulation.

The described method is applied on a test geometry consisting of plate aligned in the printing plane with a cylinder on its upper surface orientated in stacking direction, Fig. 5. On this geometry, various drill holes are added to evaluate the warpage by the meaning of ovalisation.

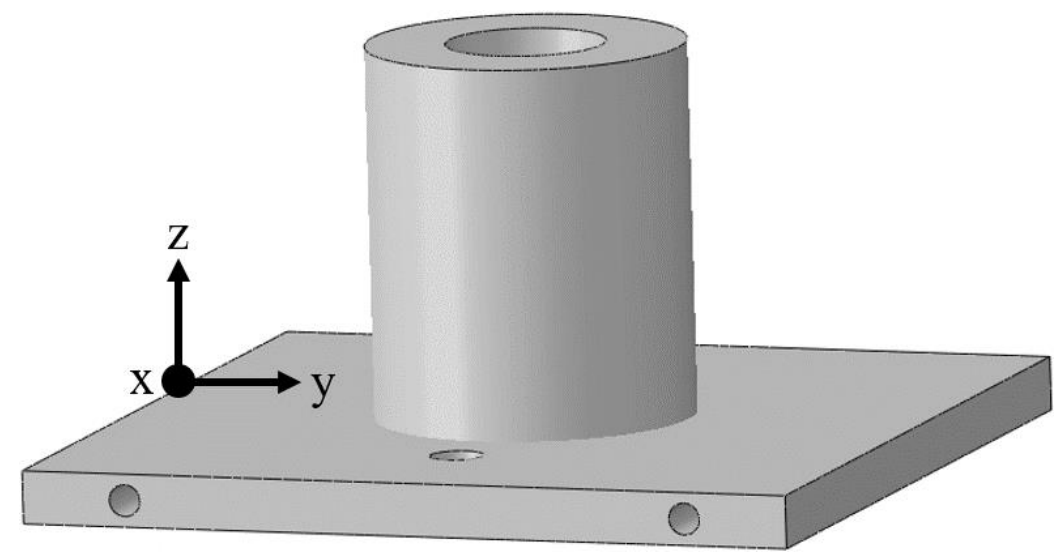

Figure 5: Test geometry. 


\section{RESULTS}

\subsection{Material modelling}

The simulation results in Fig. 6 show all nine elastic parameters required for orthotropic material models as a function of porosity. It can be concluded that the RK model is sufficient to determine the elastic and the shear modulus at small porosities. The deviation of the RK model to the numerical RVE approach is growing with increasing porosity.

The values of elastic moduli at final minimum porosity level are in good agreement with the results presented in [15] and [22]. This indicates that it is reasonable to consider the connected void channels as the predominant influencing factor on the elastic properties. Looking closer at the results, the predicted RVE elastic modulus is lower than the RK modulus in stacking direction (E3) and higher in the printing plane's directions (E1, E2). The reason for this is that the RK model assumes randomly distributed voids leading to an isotropic behaviour, whereas the RVE approach is based on channel-shaped cavities affecting the elastic properties different depending on the direction. The same applies to the Poisson ratios. The influence of the local arrangement of porosity is also visible in the shear modulus behaviour. Therefore, it is reasonable to consider the orthotropic material behaviour in the subsequent simulations. As the RK model does not cover anisotropic effects, the RK model is not recommended at higher porosity levels.
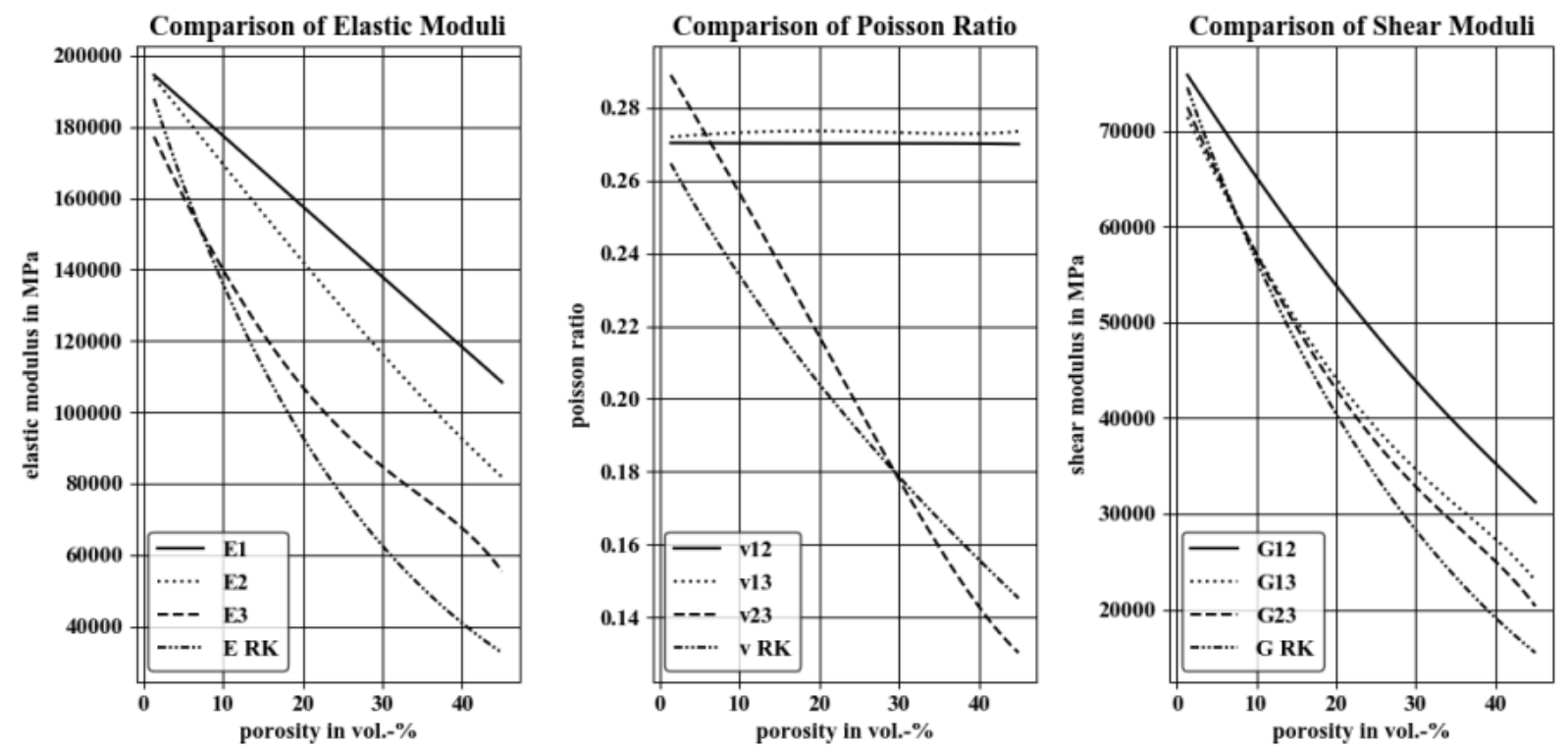

Figure 6: Comparison of elastic material parameters behaviour.

\subsection{Shrinkage prediction}

Despite the large deformation during the sinter-induced shrinkage, the simulation converged smoothly, and the global shrinkage matches the target value, the total differences between start and endpoint are shown in Fig. 7 c. This confirms the applicability of our thermomechanical approach to model the sinter-induced shrinkage. In addition, the simulation considers the non-uniform deformation of the part due to the anisotropic shrinkage behaviour. To analyse this effect, the inner shape of the drill holes, orientated in z-y direction, is plotted before and after the sintering process and its circularity is compared. Fig. 7 a clearly shows the transition from a circular to an elliptical contour during sintering. Furthermore, the distribution and the location of the mechanical stress provide additional information about the potential weak points of the design. As shown in Fig. $7 \mathrm{~b}$, the maximum stresses occur at the transition from cylinder to plate, which is a plausible result for this geometric design. 
Due to our simple thermomechanical approach, the shrinkage within the printing plane is always identical regardless its position in stacking direction. The slight influence of the position in stacking direction on the shrinkage within the printing plane as reported in [10] is not covered here.
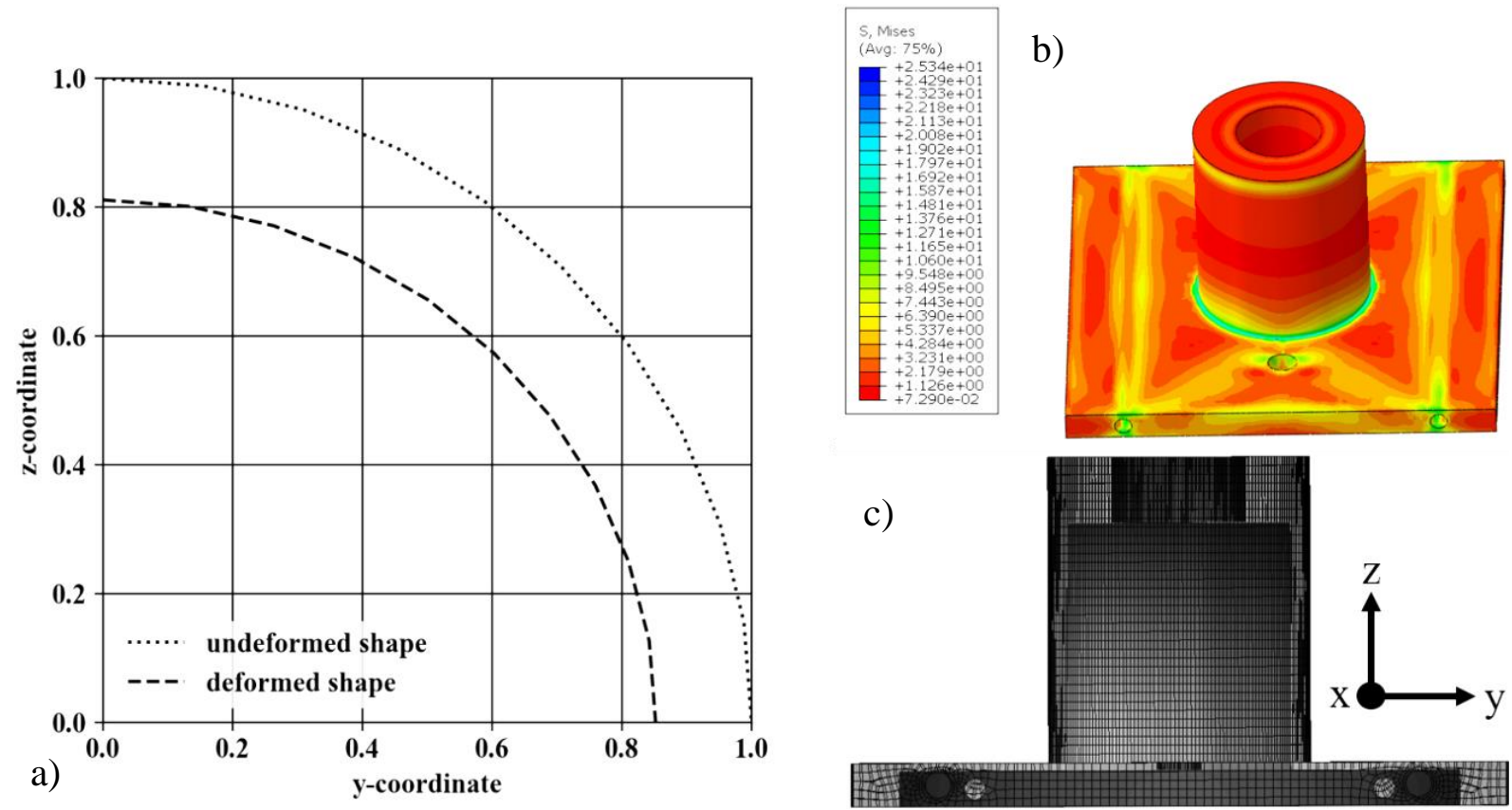

Figure 7: Results of the shrinkage simulation: a) ovalisation of circular holes, b) stress distribution and c) overlay plot.

\section{CONCLUSION}

The fabrication of metallic parts by EAM methods has the potential to become a widely used process due to its advantages over classical powder-based AM techniques. High shrinkage rates are crucial for the design step. Conventional methods for the simulation of the sintering process often demand a non-reasonable experimental effort. The approach presented here describes the sintering process based on a simple transient thermomechanical analogous model, which is already completely defined by the specification of the direction-dependent shrinkage, reducing the amount of needed material data immensely. Even though the results of a viscoplastic analysis might be more accurate, our approach leads to a reasonable prediction of shrinkage, warpage and local stress concentrations, which confirms its applicability. In future work, the simulation model will be compared with experimental results to further validate the modelling approach and confirm its applicability. Furthermore, we want to conduct design guidelines for the minimisation of internal stresses during the sintering process based on the simulation results.

\section{REFERENCES}

[1] Peko, I.; Gjeldum, N.; Bilić, B. (2018). Application of AHP, fuzzy AHP and PROMETHEE method in solving additive manufacturing process selection problem, Tehnički vjesnik Technical Gazette, Vol. 25, No. 2, 453-461, doi:10.17559/TV-20170124092906

[2] Ramadami, R.; Belsak, A.; Kegl, M.; Predan, J.; Pehan, S. (2018). Topology optimization based design of lightweight and low vibration gear bodies, International Journal of Simulation Modelling, Vol. 17, No. 1, 92-104, doi:10.2507/IJSIMM17(1)419

[3] Deckers, J.; Kruth, J.-P.; Cardon, L.; Shahzad, K.; Vleugels, J. (2013). Densification and geometrical assessments of alumina parts produced through indirect selective laser sintering of 
alumina-polystyrene composite powder, Strojniski vestnik - Journal of Mechanical Engineering, Vol. 59, No. 11, 646-661, doi:10.5545/sv-jme.2013.998

[4] Deese, K.; Geilen, M.; Rieg, F. (2018). A two-step smoothing algorithm for an automated product development process, International Journal of Simulation Modelling, Vol. 17, No. 2, 308-317, doi:10.2507/IJSIMM17(2)437

[5] Vukelic, D.; Agarski, B.; Budak, I.; Simunovic, G.; Buchmeister, B.; Jakovljevic, Z.; Tadic, B. (2019). Eco-design of fixtures based on life cycle and cost assessment, International Journal of Simulation Modelling, Vol. 18, No. 1, 72-85, doi:10.2507/IJSIMM18(1)463

[6] Bourell, D. L. (2016). Perspectives on additive manufacturing, Annual Review of Materials Research, Vol. 46, 18 pages, doi:10.1146/annurev-matsci-070115-031606

[7] Rane, K.; Strano, M. (2019). A comprehensive review of extrusion-based additive manufacturing processes for rapid production of metallic and ceramic parts, Advances in Manufacturing, Vol. 7, No. 2, 155-173, doi:10.1007/s40436-019-00253-6

[8] Gonzalez-Gutierrez, J.; Godec, D.; Kukla, C.; Schlauf, T.; Burkhardt, C.; Holzer, C. (2017). Shaping, debinding and sintering of steel components via fused filament fabrication, Proceedings of the $16^{\text {th }}$ International Scientific Conference on Production Engineering, 99-104

[9] Scime, L.; Wolf, S. D.; Beuth, J.; Mrdjenovich, S.; Kelley, M. (2018). Safety and workflow considerations for modern metal additive manufacturing facilities, The Journal of The Minerals, Metals \& Materials Society, Vol. 70, No. 9, 1830-1834, doi:10.1007/s11837-018-2971-4

[10] Gong, H.; Snelling, D.; Kardel, K.; Carrano, A. (2019). Comparison of stainless steel 316L parts made by FDM- and SLM-based additive manufacturing processes, The Journal of The Minerals, Metals \& Materials Society, Vol. 71, No. 3, 880-885, doi:10.1007/s11837-018-3207-3

[11] Tofail, S. A. M.; Koumoulos, E. P.; Bandyopadhyay, A.; Bose, S.; O’Donoghue, L.; Charitidis, C. (2018). Additive manufacturing: scientific and technological challenges, market uptake and opportunities, Materials Today, Vol. 21, No. 1, 22-37, doi:10.1016/j.mattod.2017.07.001

[12] Gonzalez-Gutierrez, J.; Cano, S.; Schuschnigg, S.; Kukla, C.; Sapkota, J.; Holzer, C. (2018). Additive manufacturing of metallic and ceramic components by the material extrusion of highlyfilled polymers: a review and future perspectives, Materials, Vol. 11, No. 5, Paper 840, 36 pages, doi: $10.3390 / \mathrm{ma} 11050840$

[13] Wu, G.; Langrana, N. A.; Rangarajan, S.; McCuiston, R.; Sadanji, R.; Danforth, S.; Safari, A. (1999). Fabrication of metal components using FDMet: fused deposition of metals, Proceedings of the Solid Freeform Fabrication Symposium, 775-782

[14] BASF 3D Printing Solutions GmbH (2018). Ultrafuse 316LX: BASF's 'Catamold ${ }^{\circledR}$ on a spool' presents opportunities for MIM producers, Powder Injection Moulding International, Vol. 12, No. 3, 83-87

[15] Jimbo, K.; Tateno, T. (2019). Shape contraction in sintering of 3D objects fabricated via metal material extrusion in additive manufacturing, International Journal of Automation Technology, Vol. 13, No. 3, 354-360, doi:10.20965/ijat.2019.p0354

[16] Hržan, M.; Šercer, M. (2019). Influence of mould wall temperature and content of recycled material on shrinkage of polymeric part, Tehnički vjesnik - Technical Gazette, Vol. 26, No. 4, 1182-1185, doi:10.17559/TV-20170713174111

[17] Sahli, M.; Djoudi, H.; Gelin, J.-C.; Barriere, T.; Assoul, M. (2018). Numerical simulation and experimental analysis of the sintered micro-parts using the powder injection molding process, Microsystem Technologies, Vol. 24, No. 3, 1495-1508, doi:10.1007/s00542-017-3533-3

[18] Gasik, M.; Zhang, B. (2000). A constitutive model and FE simulation for the sintering process of powder compacts, Computational Materials Science, Vol. 18, No. 1, 93-101, doi:10.1016/S0927$\underline{0256(00) 00090-2}$

[19] Hsu, H. J.; Lee, S. Y.; Chang, S. L.; Lo, C. H.; Lin, Y. M. (2017). Shrinkage prediction using finite element analysis and experimental validation using three-dimension slurry printing system, The International Journal of Advanced Manufacturing Technology, Vol. 91, No. 1-4, 1289-1296, doi:10.1007/s00170-016-9842-3

[20] Matsubara, S.; Tarada, K.; Kobayashi, T.; Saitou, T.; Umeda, M.; Mihara, Y.; Oide, K.; Shin, H.; Katsuda, Y. (2017). A set of constitutive functions for dried body to predict entire deformation process of ceramic products during firing, Engineering Computations, Vol. 34, No. 8, 2668-2697, doi: $\underline{10.1108 / E C-12-2016-0444}$ 
[21] Park, S. J.; Chung, S. H.; Johnson, J. L.; German, R. M. (2006). Finite element simulation of liquid phase sintering with tungsten heavy alloys, Materials Transactions, Vol. 47, No. 11, 2745-2752, doi:10.2320/matertrans.47.2745

[22] Damon, J.; Dietrich, S.; Gorantla, S.; Popp, U.; Okolo, B.; Schulze, V. (2019). Process porosity and mechanical performance of fused filament fabricated 316L stainless steel, Rapid Prototyping Journal, Vol. 25, No. 7, 1319-1327, doi:10.1108/RPJ-01-2019-0002

[23] Koval'chenko, M. S. (2003). Elasticity and viscosity of isotropic porous materials, Powder Metallurgy and Metal Ceramics, Vol. 42, No. 1-2, 81-87, doi:10.1023/A:1023955418754

[24] Ramakrishnan, N.; Arunachalam, V. S. (1990). Effective elastic moduli of porous solids, Journal of Material Sciences, Vol. 25, No. 9, 3930-3937, doi:10.1007/BF00582462

[25] Herakovich, C. T.; Baxter, S. C. (1999). Influence of pore geometry on the effective response of porous media, Journal of Material Sciences, Vol. 34, No. 7, 1595-1609, doi:10.1023/ A: 1004528600213

[26] Choren, J. A.; Heinrich, S. M.; Silver-Thorn, M. B. (2013). Young's modulus and volume porosity relationships for additive manufacturing applications, Journal of Material Sciences, Vol. 48, No. 15, 5103-5112, doi:10.1007/s10853-013-7237-5

[27] Elruby, A. Y.; Nakhla, S. (2019). Extending the Ramberg-Osgood relationship to account for metal porosity, Metallurgical and Materials Transactions A, Vol. 50, No. 7, 3121-3131, doi: $\underline{10.1007 / \mathrm{s} 11661-019-05236-7}$ 